\title{
A Measurement System for the Position and Phase Errors of the Elements in an Antenna Array Subject to Mutual Coupling
}

\author{
Tore Lindgren and Johan Borg \\ Department of Computer Science, Electrical and Space Engineering, Luleå University of Technology, 97187 Luleå, Sweden \\ Correspondence should be addressed to Tore Lindgren, tore.lindgren@ltu.se
}

Received 31 May 2011; Revised 17 October 2011; Accepted 31 October 2011

Academic Editor: Hoi Shun Lui

Copyright ( $) 2012$ T. Lindgren and J. Borg. This is an open access article distributed under the Creative Commons Attribution License, which permits unrestricted use, distribution, and reproduction in any medium, provided the original work is properly cited.

\begin{abstract}
When deploying large antenna arrays in arctic environments, a local measurement system may be necessary in order to ensure control over the position and phase of the individual antenna elements. In this paper, a method of estimating the position and phase of each individual antenna element in the presence of mutual coupling is presented. It uses both measurements of the scattering matrix in the array and measurements of the electric field using a minimum of four probes located in the near field of the array. Simulations show that the method gives accurate results even in the presence of noise in the measurements. The geometry of the probe-array system affects the performance significantly.
\end{abstract}

\section{Introduction}

In large antenna arrays it is important to know both the position and the active far-field radiation pattern of each antenna element. This is particularly the case in applications where knowledge about the gain and beam pointing direction is needed in absolute numbers, such as in multistatic radar systems. In the absence of coupling, the complex far-field radiation pattern can be estimated using the equivalent electric current method [1]. In many cases the mutual coupling between elements will distort the behavior of the individual elements. Also, the position of the antenna elements might not be constant over time. In this paper, a measurement system capable of determining the both the position and complex gain of the individual antenna elements in a physically and electrically large antenna array in the presence of mutual coupling is presented.

All large antenna arrays will thus be subject to significant random variations in the antenna elements' position and limited phase accuracy, which will affect the far field of the antenna array after beamforming if not taken into account. This was analyzed for an array with known position of the elements but a phase and amplitude error on each element [2] and for an array with both position and amplitude and phase errors on the elements [3]. In [4], the effect of random errors on the sidelobe level of a two-dimensional scanning array was investigated. There, analyses showed that the amplitude of the main beam decreased and that the sidelobe level increased when random errors were introduced. To obtain maximum performance, it is therefore important to be able to estimate the radiation properties of each antenna element.

The antenna array system considered in this paper is the planned EISCAT_3D incoherent scatter radar [5]. This system will consist of several sites with a baseline of 90$280 \mathrm{~km}$. Each site will host an antenna array with up to 16000 antenna elements operating in the $210-240 \mathrm{MHz}$ frequency band. Since the system will be multistatic and each antenna array will have narrow beams, it is crucial to have accurate control over the pointing direction and shape of the beam from the array. It has been found that the maximum acceptable delay error between any two antenna elements is 160 ps [6]. This limit includes all errors such as jitter in the local oscillator and in the analog-to-digital converter (ADC), errors introduced in the clock distribution system, changes in the phase of the far-field pattern of the antenna elements, and changes in the physical position of the antenna elements. Furthermore, the array will operate 
in remote, arctic, locations. This fact, in combination with the large physical size of the system, introduces some extra requirements on the system.

(i) Any cables used in the system, for example, clock distribution, will be subject to large temperature variations. This will introduce additional, potentially unacceptable, errors in the timing of the individual front-ends. Several methods of mitigating this problem have been proposed, see for example, [7].

(ii) The performance of the antenna elements might also degrade due to snowfall [8]. The measurement system presented in [1] is one means of mitigating these effects.

(iii) In the area where the antenna array will be located the ground will freeze every winter. This might induce ground movements which means that the position of the antenna elements might not be constant. It is therefore important to have the capability of accurately measure the position of each antenna element.

(iv) The field of view of the array will be large (nearly hemispherical). It is therefore necessary to be able to estimate the performance of the array in all directions.

(v) Although the system is expected to operate continuously and thus requires continuous monitoring, the time scales relevant to the changes in performance are relatively slow, on the orders of minutes to years. The required update rate of the measurement system is therefore not likely a problem.

Several measurement methods capable of solving at least parts of the problems outlined above can be found in the literature. Traditionally, the performance of large aperture antennas is measured using radio sources such as quasars or distant galaxies [9]. The same approach has also been studied using satellite-based transmitter [10]. Although both of these methods are capable of accurately measuring both the beam shape and pointing direction they are limited in field view to essentially one direction at a given time. Several distant radio sources exist covering the entire sky, making the former approach more attractive. The signal strengths involved are however generally too weak to enable calibration of the individual antenna elements. The simplicity of the method makes it an attractive complement to a local measurement system.

In the planned antenna it will be possible to directly measure the scattering matrix of the array, which can be used to find the impedance and admittance matrices. This information has been used previously to estimate the active radiation pattern of the antenna elements, see for example, $[11,12]$. In both these cases the position of the elements was assumed to be known. In the latter paper, a small number of calibration sources ( 9 calibration elements is sufficient for a 10000-element array) was used to estimate the coupling. In [13] a similar calibration scheme was presented intended for the Square Kilometer Array. This method made use of an initial offline calibration using far-field sources and an online calibration method based on a monitoring system proposed in [14]. A more recent paper present a method for estimation of the coupling matrix, the elements' radiation pattern and the phase center of the array [15]. This method uses only probes located in the far-field of the array. Thus far, no method have been presented which is capable of estimating both the active far-field radiation pattern and the phase centers of the antenna elements in an array subject to mutual coupling. The method proposed in the following sections fulfills this requirement using a minimum of four probes located in the near-field of the antenna array. The feasibility of the method is demonstrated using simulations in which the dilution of precision is used to assess the geometrical effects.

\section{Measurement System}

In order to determine the position and phase error of each individual antenna element in an array subject to coupling, the measurement procedure is divided into two parts. Firstly, the full scattering matrix is measured and secondly, the array is illuminated by at least four probes in order to obtain the position and phase of each element. These two measurements are described in the subsections below.

2.1. The Mutual Coupling between Antenna Elements. The frequency domain behavior of any linear electric network can be described completely in terms of a matrix relating complementary quantities at all ports [16]. One such description is to express the voltages $\mathbf{V}$ at all ports terms of the currents flowing into all ports I as

$$
\mathbf{V}=\mathbf{Z I} \text {, }
$$

where $\mathbf{Z}$ is the frequency-dependent impedance parameter matrix. Other representations include the admittance matrix

$$
\mathbf{Y}=\mathbf{Z}^{-1} \text {, }
$$

which relates short-circuit currents to applied voltages, and the scattering parameter matrix $\mathbf{S}$ which describes the network in terms of incident to and reflected/transmitted electrical waves of some characteristic impedance. The $\mathbf{S}$ matrix is related to the impedance parameter matrix $\mathbf{Z}$ of the same network as

$$
\mathbf{Z}=z_{\text {ref }}(\mathbf{U}+\mathbf{S})(\mathbf{U}-\mathbf{S})^{-1},
$$

where $\mathbf{U}$ is the identity matrix.

For this paper, we assume that a scattering-parameter model of the interaction between all antennas within the array is measured by applying an electrical stimulus from a source of a suitable impedance (e.g., $50 \Omega$ ) at one antenna at a time, and observing both the reflected wave at the driven port and also the transmitted signals arriving at all other receivers of the arrays. It should be noted that errors in actual impedance of the receiver front-end or errors in the cable-lengths used between front-end and antenna can be calibrated out based on measurements on reference 
open/short/match elements at the time of installation. In Chapter 3 in [17] details about an architecture capable of performing these measurements is presented. Here it is assumed that the conditions stated in [18] are fulfilled. The method was also recently analyzed rigorously [19].

2.2. The Electric Field Produced by an Antenna Array. The radiation properties of an antenna is described using amplitude, phase, and polarization. In order to measure this a number of probes will be necessary. A comprehensive description of antenna measurement procedures can be found in [20].

The current on an antenna located at a point $\mathbf{r}$ is proportional to the electric field $\mathbf{E}$ in the same point with an angular dependence $\mathbf{G}(-\hat{\mathbf{r}})$ which yields

$$
I=a \mathbf{G}(\hat{\mathbf{r}}) \cdot \mathbf{E}(\mathbf{r}) .
$$

In this application, the main interest is in the position and time errors of the system. The relevant property to measured is therefore the phase of the far-field radiation pattern of the antenna elements. For these reasons it is crucial that the probe is well characterized in terms of position, phase, and polarization, that is, $\mathbf{G}$ and $\mathbf{r}$ are known with sufficient accuracy. In order to estimate the position and phase error of each antenna element at least four probes are needed. Furthermore, these probes needs to be well synchronized with each other.

\section{The Complex Amplitude and Position}

In this section the calculations associated with the electric field produced by the antenna array and the mutual coupling between the elements in the array are presented. In Section 3.1 both these are used to estimate the contribution of each antenna element to the complex field produced by the array and in Section 3.2 the phase of this contribution is compared with the expected value (i.e., the nominal geometric range between the probe and the antenna element). This relates directly to the geometric range and the position and phase error of each antenna element can then be estimated using trilateration in the same way as is done in, for example, GPS receivers.

3.1. The Electric Field. The electric field $\mathbf{E}$ at a point $\mathbf{r}$ in the radiative near-field of an antenna consisting of a perfect electric conductor can be calculated using

$$
\mathbf{E}(\mathbf{r})=-j \omega \mathbf{A}(\mathbf{r})
$$

with

$$
\mathbf{A}(\mathbf{r})=\mu \int_{S^{\prime}} \frac{e^{-j \omega r}}{4 \pi r} \mathbf{J}\left(\mathbf{r}^{\prime}\right) d S^{\prime},
$$

where $\omega$ is the angular frequency, $\mu$ is the permeability of the medium, $S^{\prime}$ is the surface of the antenna, $\mathbf{J}\left(\mathbf{r}^{\prime}\right)$ is the electric current at the point $\mathbf{r}^{\prime}$ on the antenna, and $r=\left|\mathbf{r}-\mathbf{r}^{\prime}\right|$.
In the far-field region, the magnetic vector potential $\mathbf{A}$ is approximated by

$$
\mathbf{A}(\mathbf{r}) \approx \frac{\mu e^{-j k r}}{4 \pi r} \mathbf{G}(\widehat{\mathbf{r}})
$$

where

$$
\mathbf{G}(\hat{\mathbf{r}})=\int_{S^{\prime}} \mathbf{J}\left(\mathbf{r}^{\prime}\right) e^{j k r^{\prime} \cos \psi} d S^{\prime}
$$

is the far field radiation pattern of the antenna and $\psi$ is the angle between the vectors $\mathbf{r}$ and $\mathbf{r}^{\prime}$.

The current distribution can be expanded using basis functions according to

$$
\mathbf{J}\left(\mathbf{r}^{\prime}\right)=\sum_{n=1}^{N} \alpha_{n} \mathbf{f}_{n}\left(\mathbf{r}^{\prime}\right)
$$

where $\alpha_{n}$ are constants, $\mathbf{f}_{n}\left(\mathbf{r}^{\prime}\right)$ are suitable basis functions, and $N$ is the total number of basis functions. The electric field in (5) can then be written as

$$
\mathbf{E}(\mathbf{r})=\frac{e^{-j k r+\phi}}{r} \sum_{n=1}^{N} \alpha_{n} \mathbf{G}_{n}^{\prime}(\widehat{\mathbf{r}}),
$$

where $\phi$ is an unknown phase shift, $\widehat{\mathbf{r}}=\left(\mathbf{r}-\mathbf{r}^{\prime}\right) / r$, and

$$
\mathbf{G}_{n}^{\prime}(\widehat{\mathbf{r}})=\frac{\mu}{4 \pi} \int_{S^{\prime}} \mathbf{f}_{n}\left(\mathbf{r}^{\prime}\right) e^{j k r^{\prime} \cos \psi} d S^{\prime} .
$$

If the antenna elements in the array are simple it will be sufficient to use one basis function only, for example, a sinusoid if half wave dipoles are used. In this case the parameter $\alpha_{n}$ will be the current at the input port of the antenna. Then (10) reduces to

$$
\mathbf{E}(\mathbf{r})=\frac{e^{-j k r+\phi}}{r} I^{\prime}(\hat{\mathbf{r}}) .
$$

The current induced in the probe antenna $p$ from antenna element $q$ will thus be

$$
I_{p}=\frac{e^{-j k r_{p q}+\phi_{q}}}{r_{p q}} I_{q} \mathbf{G}_{q}\left(\widehat{\mathbf{r}}_{p q}\right) \cdot \mathbf{G}_{p}\left(-\widehat{\mathbf{r}}_{p q}\right),
$$

where $\mathbf{G}_{q}\left(\hat{\mathbf{r}}_{p q}\right)$ is the far field radiation pattern of antenna element $q$ in the direction towards the probe $p$ and $\mathbf{G}_{p}\left(-\hat{\mathbf{r}}_{p q}\right)$ is the far field radiation pattern of the probe $p$ in the direction towards the antenna element $q$.

For a set of measurements of different antenna elements in the array (13) can be written as

$$
\mathbf{I}_{a}=\left(\mathbf{G}_{q}\left(\widehat{\mathbf{r}}_{p q}\right) \cdot \mathbf{G}_{p}\left(-\widehat{\mathbf{r}}_{p q}\right)\right) \mathbf{R}_{p q} \mathbf{I}_{a q},
$$

where $\mathbf{I}_{a}$ is a row vector containing the measured currents at the probe for each measurement $a$,

$$
\mathbf{R}_{q}=\frac{e^{-j\left(k r_{p q}+\phi_{q}\right)}}{r_{p q}}
$$


and $\mathbf{I}_{a q}$ is a matrix where each row defines the current distribution over the array for measurement $a$. This can now be related to the admittance matrix in (3) which yields

$$
\mathbf{I}_{a}=\left(\mathbf{G}_{q}\left(\widehat{\mathbf{r}}_{p q}\right) \cdot \mathbf{G}_{p}\left(-\widehat{\mathbf{r}}_{p q}\right)\right) \mathbf{R}_{p q} \mathbf{Y}_{q s} \mathbf{V}_{q a},
$$

where $\mathbf{Y}_{q s}$ is the admittance matrix of the array and $\mathbf{V}_{q a}$ is the excitation of the array with each column containing the excitation for a particular measurement $a$.

Although any type of excitation scheme could be used it is most convenient to excite the antenna elements one at a time with equal voltages. In this case the matrix $\mathbf{V}_{q a}$ will be equal to the identity matrix. Due to reciprocity, this will also be the case if the probes are used in transmitting mode (even though different voltages will be seen at each element). In (16), the unknown to be solved for is the term $\mathbf{R}_{p q}$ which contains the information about the distance and electrical phase errors of each antenna element. This is given by

$$
\mathbf{R}_{p q}=\frac{\mathbf{I}_{a} \mathbf{Y}_{q s}^{-1}}{\left(\mathbf{G}_{q}\left(\hat{\mathbf{r}}_{p q}\right) \cdot \mathbf{G}_{p}\left(-\hat{\mathbf{r}}_{p q}\right)\right)}
$$

Inserting (3) into (17) then yields

$$
\mathbf{R}_{p q}=\frac{z_{\mathrm{ref}} \mathbf{I}_{a}\left(U+S_{q s}\right)\left(U-S_{q s}\right)^{-1}}{\left(\mathbf{G}_{q}\left(\hat{\mathbf{r}}_{p q}\right) \cdot \mathbf{G}_{p}\left(-\hat{\mathbf{r}}_{p q}\right)\right)} .
$$

The procedure outlined above is repeated for each probe in order to get a full set of measurements to be used when solving for the position of the antenna elements.

3.2. The Position Solution. The present measurement system assumes that the AUT's approximate location $\mathbf{r}_{0}^{\prime}$ and the nominal radiation pattern $\mathbf{G}_{0}^{\prime}$ are known. Furthermore, it is assumed that the antenna elements are simple enough so that their individual radiation patterns only change by a complex constant (due to for example, a phase shift or displacement of the element). These assumptions enable solving for the free space path loss and phase offset, $\mathbf{R}_{p q}$, of the individual antenna elements as was shown in the previous subsection. To obtain the position and phase offset of the antenna elements the phase of $\mathbf{R}_{q}$ must be studied. This can be written as

$$
\angle \mathbf{R}_{p q}=\left\|\mathbf{r}_{p}-\mathbf{r}_{q}^{\prime}\right\|+\phi_{q}
$$

In the actual antenna array the approximate position will be known from the design of the array and the approximate clock error will be known from the clock distribution system. Thus (19) can be rewritten as

$$
\angle \mathbf{R}_{p q}=\left\|\mathbf{r}_{p}-\mathbf{r}_{q, 0}^{\prime}-\delta \mathbf{r}_{q}^{\prime}\right\|+\phi_{q, 0}+\delta \phi_{q},
$$

where $\mathbf{r}_{q, 0}^{\prime}$ and $\phi_{q, 0}$ is the approximate position and clock error, respectively, and $\delta \mathbf{r}_{q}^{\prime}$ and $\delta \phi_{q}$ are the errors.

If at least four linearly independent measurements $m$ are available (20) can be solved iteratively using the NewtonRaphson method. The procedure is well known from the satellite navigation community and a detailed derivation can be found in for example [21]. Thus, only the solution is presented here. The problem is solved iteratively and the solution can for this application be written as a system of linear equations as

$$
A_{q, i}\left[\begin{array}{l}
\delta \mathbf{r}_{q, i+1}^{\prime} \\
\delta \phi_{q, i+1}
\end{array}\right]=b_{p q, i}
$$

where $i$ is the iteration index,

$$
\begin{aligned}
A_{q, i}= & {\left[\begin{array}{ll}
\widehat{\mathbf{r}}_{q p, i} & 1
\end{array}\right] } \\
b_{p q, i}= & \angle \mathbf{R}_{p q}-\left\|\mathbf{r}_{p}-\mathbf{r}_{q, i}^{\prime}-\delta \mathbf{r}_{q, i}^{\prime}\right\| \\
& +\phi_{q, i}+\delta \phi_{q, i}+\varepsilon_{p q},
\end{aligned}
$$

where $\mathbf{r}_{q, 0}^{\prime}$ and $\phi_{q, 0}$ is the nominal position and phase offset, $\delta \mathbf{r}_{q, i}^{\prime}$ and $\delta \phi_{q, i}$ are the guesses of the errors in the nominal position and phase offset, $\varepsilon_{m q}$ is the measurement noise, and

$$
\widehat{\mathbf{r}}_{q m, i}=\frac{\mathbf{r}_{m}-\mathbf{r}_{q, i}^{\prime}-\delta \mathbf{r}_{q, i}^{\prime}}{\left\|\mathbf{r}_{m}-\mathbf{r}_{q, i}^{\prime}-\delta \mathbf{r}_{q, i}^{\prime}\right\|}
$$

is the estimated direction from the antenna element $q$ towards the probe $p$. Since the errors $\delta \mathbf{r}_{q}^{\prime}$ and $\delta \phi_{q}$ will mainly be caused by relatively slow processes such as ground movement, temperature variations, and precipitation, these can safely be assumed to be smaller than a wavelength between each measurement epoch. Thus, no integer ambiguity need to be considered. There are several estimators that can be used to solve the system of equations in (21). If the noise $\varepsilon_{m q}$ is white, Gaussian, with zero mean, the least square (LS) estimator will be a best linear unbiased estimator (BLUE), provided that the $\varepsilon_{p q}$ have equal variance for all probes. Then

$$
\left[\begin{array}{l}
\delta \mathbf{r}_{q, i+1}^{\prime} \\
\delta \phi_{q, i+1}
\end{array}\right]=\left(A_{q, i}^{T} A_{q, i}\right)^{-1} A_{q, i}^{T} b_{p q, i} .
$$

The computed $\delta \mathbf{r}_{q, i+1}^{\prime}$ and $\delta \phi_{q, i+1}$ are used to update the $\mathbf{r}_{q, i}^{\prime}$ and $\phi_{q, i}$ as well as $\hat{\mathbf{r}}_{q p, i}$. The procedure is then repeated until the solution converges.

The errors in the estimated position and phase of the antenna elements will, provided that the measurement errors are zero mean, also be zero mean. They will, however, have different variances in different directions and this will depend on the geometry of the probes. This is conveniently analyzed using the dilution of precision (DOP) which is given by the diagonal elements of

$$
D=\left(A^{T} A\right)^{-1} \text {. }
$$

For a system where the position of the probes and the antenna element are defined in a local east-north-up coordinate system and the all measurements have equal variance $\sigma$, the overall quality of the LS solution is given by

$$
\begin{aligned}
\sigma_{G} & =\sqrt{\sigma_{E}^{2}+\sigma_{N}^{2}+\sigma_{U}^{2}+\sigma_{\phi}^{2}} \\
& =\sigma \sqrt{D_{11}+D_{22}+D_{33}+D_{44}},
\end{aligned}
$$




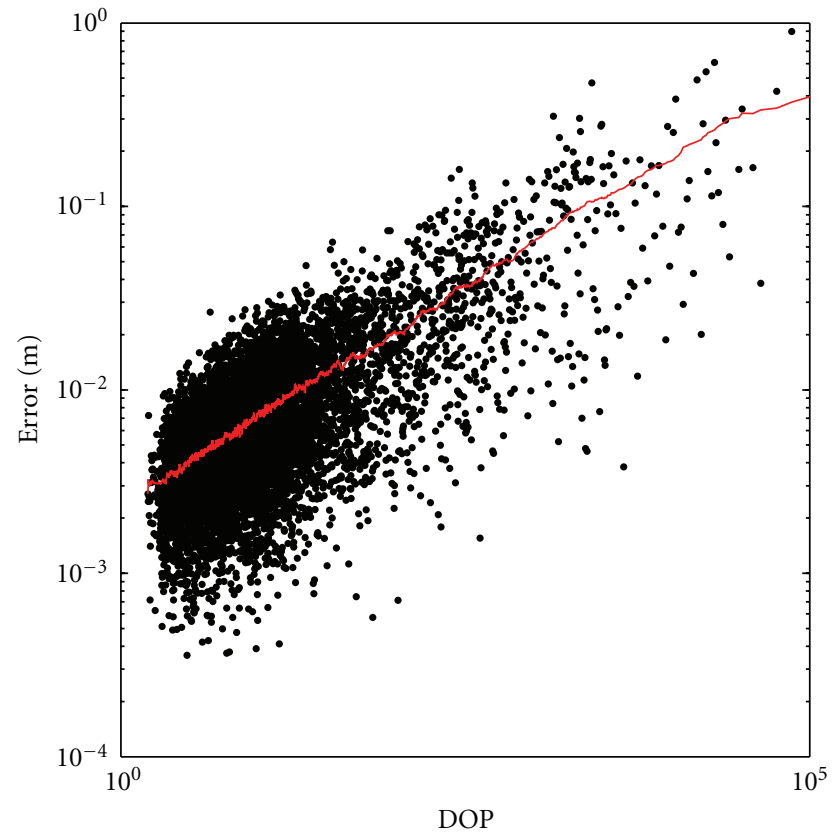

FIgURE 1: The error in the position estimation versus the dilution of precision for all antenna elements and 200 random probe configurations. The red line is a smoothed curve using a 500-point moving average.

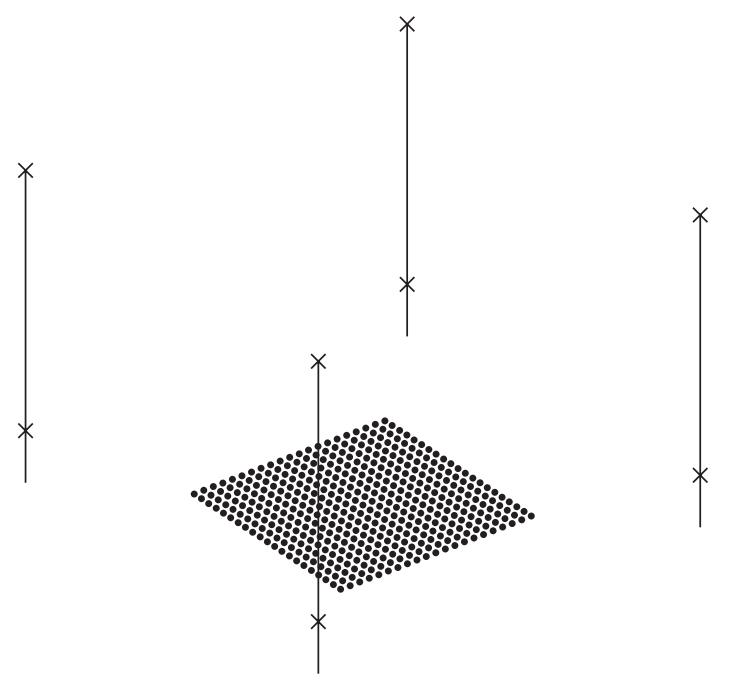

Figure 2: The setup of the antenna array system. The dots denote the antenna elements and the " $\mathrm{x}$ " are the probes.

where $\sigma_{E}^{2}, \sigma_{N}^{2}, \sigma_{U}^{2}$, and $\sigma_{\phi}^{2}$ are the variances in the east, north, up, and phase coordinates, respectively. The term $\sigma_{G} / \sigma$ is called the geometric dilution of precision (GDOP) and will be used to assess the performance of the measurement system in the next section.

\section{Results}

In this section, the performance of the measurement method outlined above is assessed using simulations. The antenna

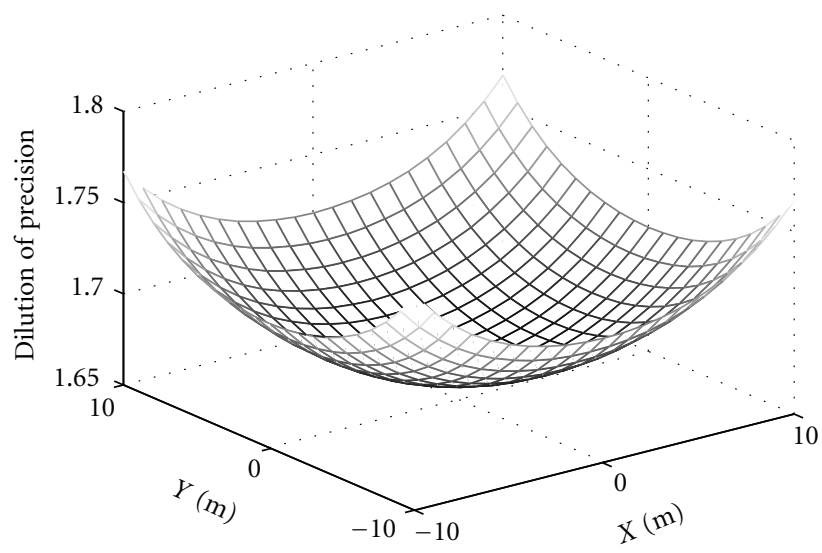

Figure 3: The geometric dilution of precision (GDOP) versus the position of the antenna element in the array.

array considered here is a 21 by 21 element array (a total of 441 elements). The elements are laid out in a square grid with a distance of one wavelength between each element. Each element is a point source with an omnidirectional radiation pattern. An operational frequency of $230 \mathrm{MHz}$ is assumed and thus the side of the array is approximately $27 \mathrm{~m}$ long.

It is assumed that each antenna element in the array couple randomly to all other antenna elements in such a way that the current $\mathbf{I}_{p q}$ in (14) is given by

$$
\mathbf{I}_{p q}=U+0.01 \alpha_{i j}
$$

where $\alpha_{i j}$ is a symmetric matrix where the elements are complex, random, and gaussian distributed (with unit variance). The measurements are assumed to have a signal to noise ratio of $30 \mathrm{~dB}$. This noise is assumed to add both to the measurements obtained by the probes and to the measurement of the coupling matrix.

In this paper, the only assumptions made about the coupling is thus that it is not dominating the behavior of the antenna. Also, there are no assumption about what type of antenna other than that it can be described with only one basis function. It is assumed that the coupling exists and that it can be measured (with measurement uncertainty) but the coupling is not determined by electromagnetic simulations in this paper. This simplification was done in order to enable the large number of simulations needed in order to get statistically significant results.

4.1. Accuracy versus Dilution of Precision. The dilution of precision assumes equal variance in all measurements. This might not be the case in this application since the distance between the antenna elements to the different probes varies with the position of the elements in the array. Also, the coupling might corrupt the measurement somewhat. The dilution of precision is here compared with the error in position and phase in order to validate the use of the dilution of precision as a measure of performance.

A total of 200 simulations was performed. The number of probes used varied between four and twelve (randomly) and 


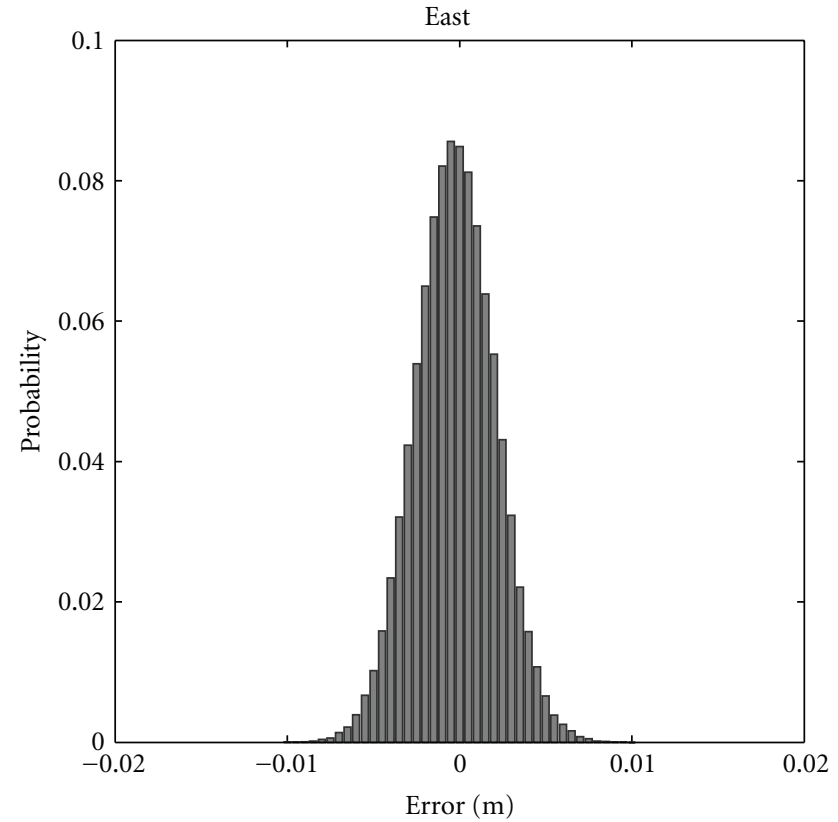

(a)

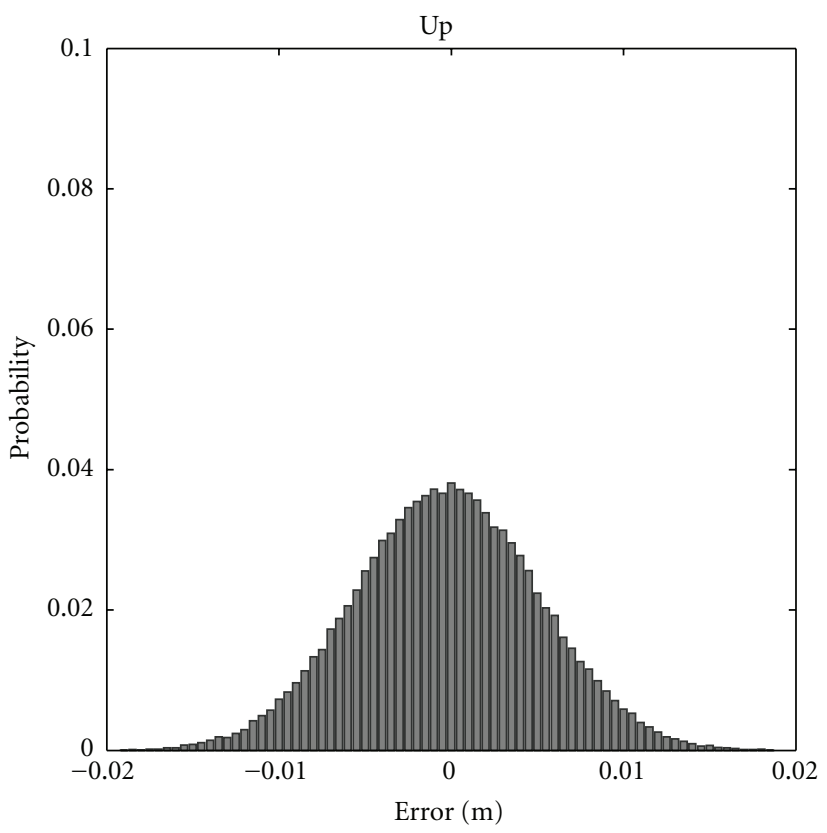

(c)

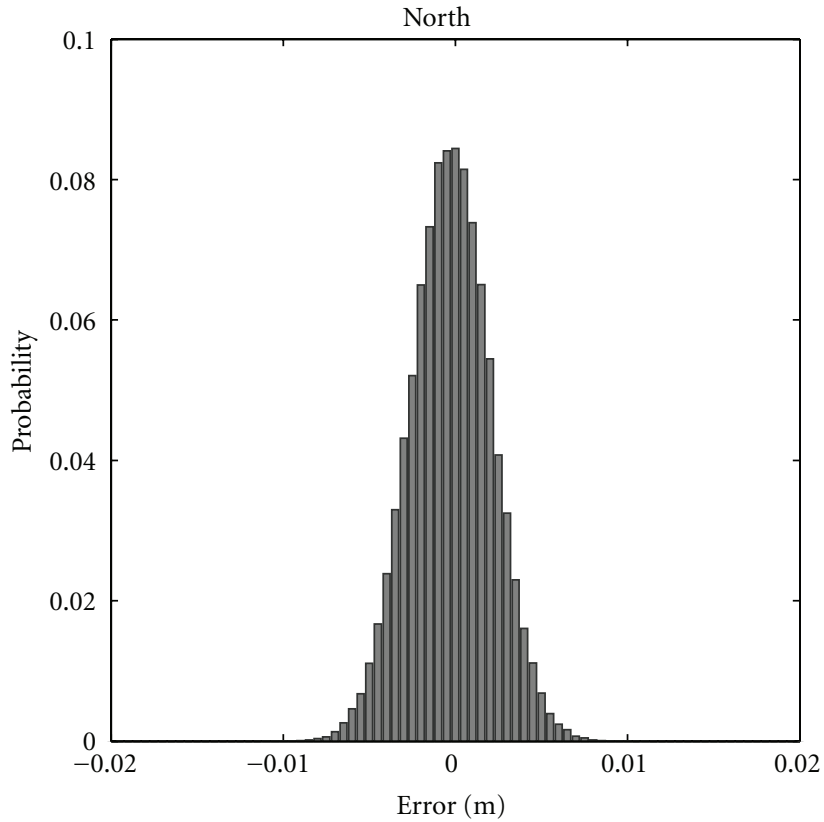

(b)

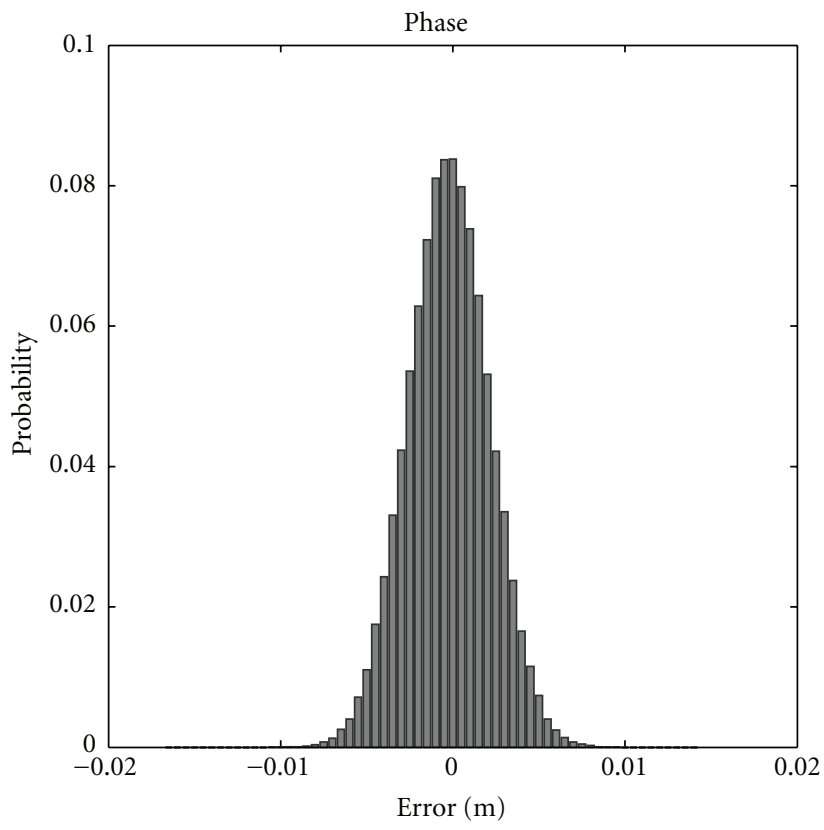

(d)

FIGURE 4: Histograms of the errors in the east, north, and up directions and the constant phase offset. The phase offset is expressed in units of meters to enable comparison with the other plots.

the probes were located at random positions in a box with sides 50-by-50-by- 25 meters. This was done in order to get a variety of DOPs for analysis.

In Figure 1 the error in the estimation of the position and phase of the antenna elements is shown versus the dilution of precision. The results for all antenna elements and all probe configurations are shown (a total of 88200 data points). The red line in the figure is a smoothed curve using a 500-point moving average. It can be seen that the average error is proportional to the DOP, at least for the lower values of DOP. For an actual implementation, a DOP of less than 10 should be expected.

4.2. Sample Measurement System. The performance of a sample measurement system was analyzed in order to obtain a more realistic performance estimate for this method. A total of eight probes is used and these are located on four different towers with east-north coordinates $(-20,-20)$, 
$(-20,20),(20,-20)$, and $(20,20)$. The probes are at height $5 \mathrm{~m}$ and $30 \mathrm{~m}$ above the ground. The layout of the antenna array and the probes is shown in Figure 2.

The GDOP of the measurement system is shown in Figure 3 for different locations in the array. It can be seen that, for this particular geometry, the performance is slightly reduced towards the edges of the array. This is expected since the probes will be more evenly spread out around elements in the center of the array, and thus the columns of $A_{q, i}$ in (21) will be closer to orthogonal here.

Since measurement uncertainty due to noise in the received signals add both to the probe measurements and the measurement of the coupling matrix, it is relevant to do a statistical evaluation of the performance. The results are shown in Figure 4. A total of 200 simulations were performed. The errors in all the antenna elements are shown in the same figure even though these errors can be expected to have slightly different distributions due to the fact that the DOP changes over the array as seen in Figure 3. The electrical phase offset of the antenna elements shown in the lower right plot in Figure 4 are expressed in meters to enable a comparison between the errors in the different directions. An error of $0.01 \mathrm{~m}$ corresponds to a phase error of approximately $3^{\circ}$. All errors have a mean of nearly 0 . The standard deviations are for the errors in the east and north directions $2.3 \mathrm{~mm}$, for the up direction $5.3 \mathrm{~mm}$, and for the phase offset $2.4 \mathrm{~mm}$. The performance is thus significantly poorer in the up-directions compared to the other directions. This is due to the fact that the probes are all located in one hemisphere.

\section{Discussion and Conclusions}

A measurement system capable of estimating both the position and phase of the antenna elements in the presence of mutual coupling has been presented. The system requires measurements of the full-coupling matrix and electric field measurements from at least four probes. The method has been demonstrated using simulations of a dipole array with random coupling between the elements.

The measurement method discussed here is mainly relevant for antenna arrays which are both electrically and physically large. This is due to the fact that smaller array are not affected to the same extent by temperature and precipitation related factors, which are expected to be a major error source in the EISCAT_3D system.

In this paper, a number of simplifying assumptions have been made. Although the feasibility of the measurement method has been demonstrated, further studies are needed before the method can be implemented in an actual system. The main question remaining is how the method works with realistic antennas and all imperfections, such as a poorly conducting ground plane and multipath effects. More rigorous simulations and measurements will be needed in order to test these issues. Also, the method here assumes simple antenna elements which can be described using only one basis function. More complex antenna elements will in general require a larger number of probes. How to make these adjustments are clear areas which require further studies.

\section{References}

[1] T. Lindgren, J. Ekman, and S. Backén, "A measurement system for the complex far-field of physically large antenna arrays under noisy conditions utilizing the equivalent electric current method," IEEE Transactions on Antennas and Propagation, vol. 58, no. 10, pp. 3205-3211, 2010.

[2] J. Ruze, "The effect of aperture errors on the antenna radiation pattern," Il Nuovo Cimento, vol. 9, supplement 3, pp. 364-380, 1952.

[3] E. N. Gilbert and S. P. Morgan, "Optimum design of directive antenna arrays subject to random variations," Bell System Technical Journal, vol. 34, pp. 637-663, 1955.

[4] R. E. Elliot, "Mechanical and electrical tolerances for twodimensional scanning antenna arrays," IEEE Transactions on Antennas and Propagation, vol. 6, no. 1, pp. 114-120, 1958.

[5] G. Wannberg, H. Andersson, R. Behlke et al., "EISCAT_3D-a next-generation European radar system for upper atmosphere and geospace research," Radio Science Bulletin, no. 332, pp. 75$88,2010$.

[6] G. Stenberg, J. Borg, J. Johansson, and G. Wannberg, "Simulation of post-ADC digital beam-forming for large area radar receiver arrays," in Proceedings of the IEEE International RF and Microwave Conference, Putra Jaya, Malaysia, September 2006.

[7] M. Morimoto, "New calibration method for large aerial arrays," Electronics Letters, vol. 1, no. 7, pp. 192-193, 1965.

[8] T. Lindgren and J. Ekman, "Performance of a Yagi antenna during snowfall," in Proceedings of the International Symposium on Antennas and Propagation, Taipei, Taiwan, October 2008.

[9] P. G. Smith, "Measurement of the complete far-field pattern of large antennas by radio-star sources," IEEE Transactions on Antennas and Propagation, vol. 14, no. 1, pp. 6-16, 1966.

[10] P. Talaga, "Measurement of a large antenna using a spacecraft as a receiver," IEEE Transactions on Antennas and Propagation, vol. 38, no. 6, pp. 883-888, 1990.

[11] H. M. Aumann, A. J. Fenn, and F. G. Willwerth, "Phased array antenna calibration and pattern prediction using mutual coupling measurements," IEEE Transactions on Antennas and Propagation, vol. 37, no. 7, pp. 844-850, 1989.

[12] A. Agrawal and A. Jablon, "A calibration technique for active phased array antennas," in Proceedings of the IEEE International Symposium on Phased Array Systems and Technology, pp. 223-228, Laramie, Wyo, USA, October 2003.

[13] G. A. Hampson and A. B. Smolders, "A fast and accurate scheme for calibration of active phased-array antennas," in Proceedings of the IEEE Antennas and Propagation Society International Symposium, vol. 2, pp. 1040-1043, August 1999.

[14] K. M. Lee, R. S. Chu, and S. C. Liu, "A Built-in performancemonitoring/fault isolation and correction (PM/FIC) system for active phased-array antennas," IEEE Transactions on Antennas and Propagation, vol. 41, no. 11, pp. 1530-1540, 1993.

[15] M. Mowlér, B. Lindmark, E. G. Larsson, and B. Ottersten, "Joint estimation of mutual coupling, element factor, and phase center in antenna arrays," EURASIP Journal on Wireless Communications and Networking, vol. 2007, Article ID 30684, 9 pages, 2007.

[16] K. Kurokawa, "Power waves and the scattering matrix," IEEE Transactions on Microwave Theory and Techniques, vol. 13, no. 2, pp. 194-202, 1965. 
[17] J. Johansson, G. Johansson, J. Borg, M. Larsmark, and T. Lindgren, "EISCAT_3D receiver/antenna subsystem report," Tech. Rep., EISCAT Scientific Association, 2009.

[18] A. T. de Hoop, "The N-port receiving antenna and its equivalent electrical network," Philips Research Report, vol. 30, pp. 302-315, 1975.

[19] J. W. Wallace and R. Mehmood, "On the accuracy of equivalent circuit models for multi-antenna systems," IEEE Transactions on Antennas and Propagation, no. 99, p. 1, 2011.

[20] “IEEE standard test procedures for antennas," ANSI/IEEE Std 149-1979, 1979.

[21] P. Misra and P. Enge, Global Positioning System: Signals, Measurements and Performance, Ganga-Jamuna, Lincoln, Mass, USA, 2nd edition, 2006. 

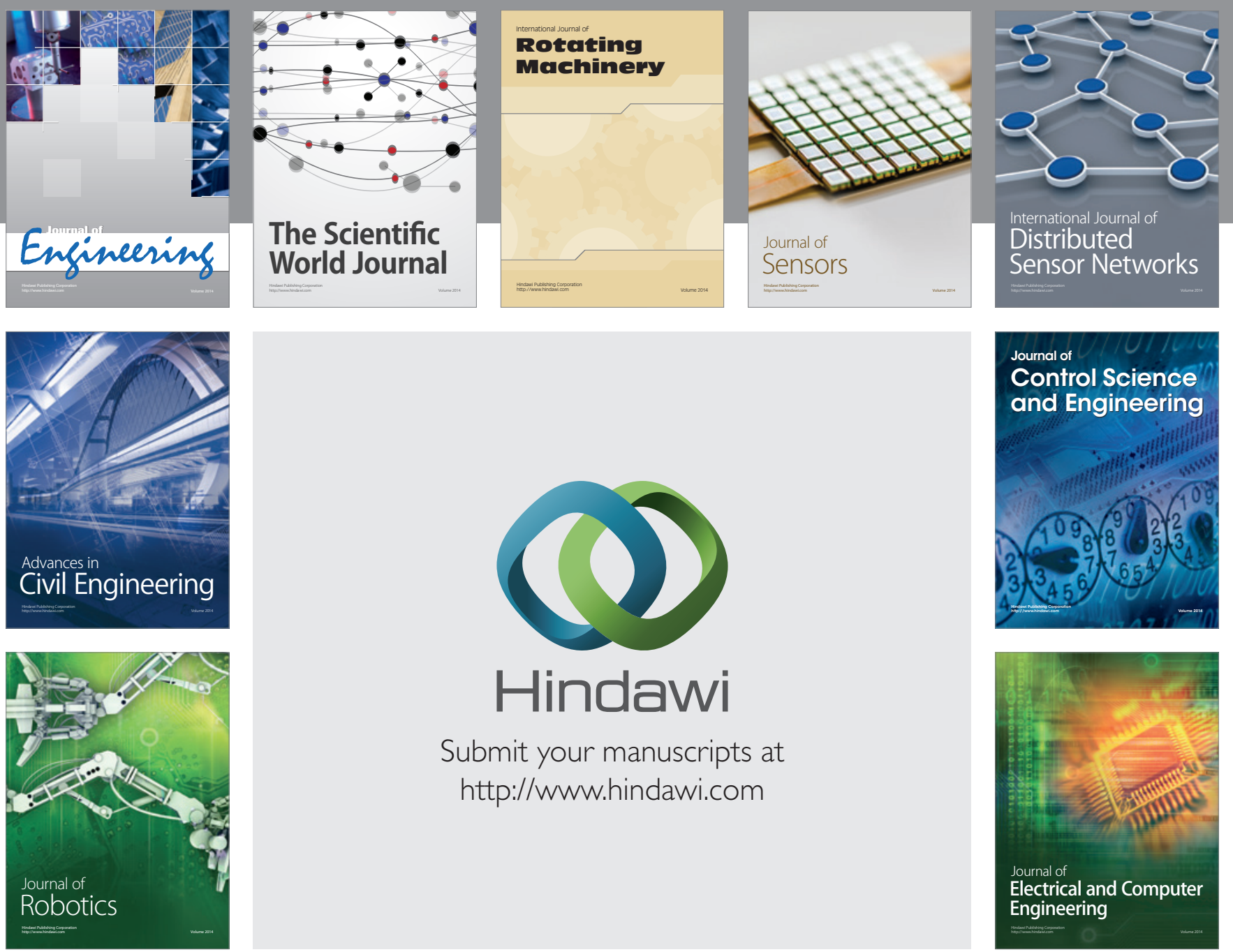

Submit your manuscripts at

http://www.hindawi.com
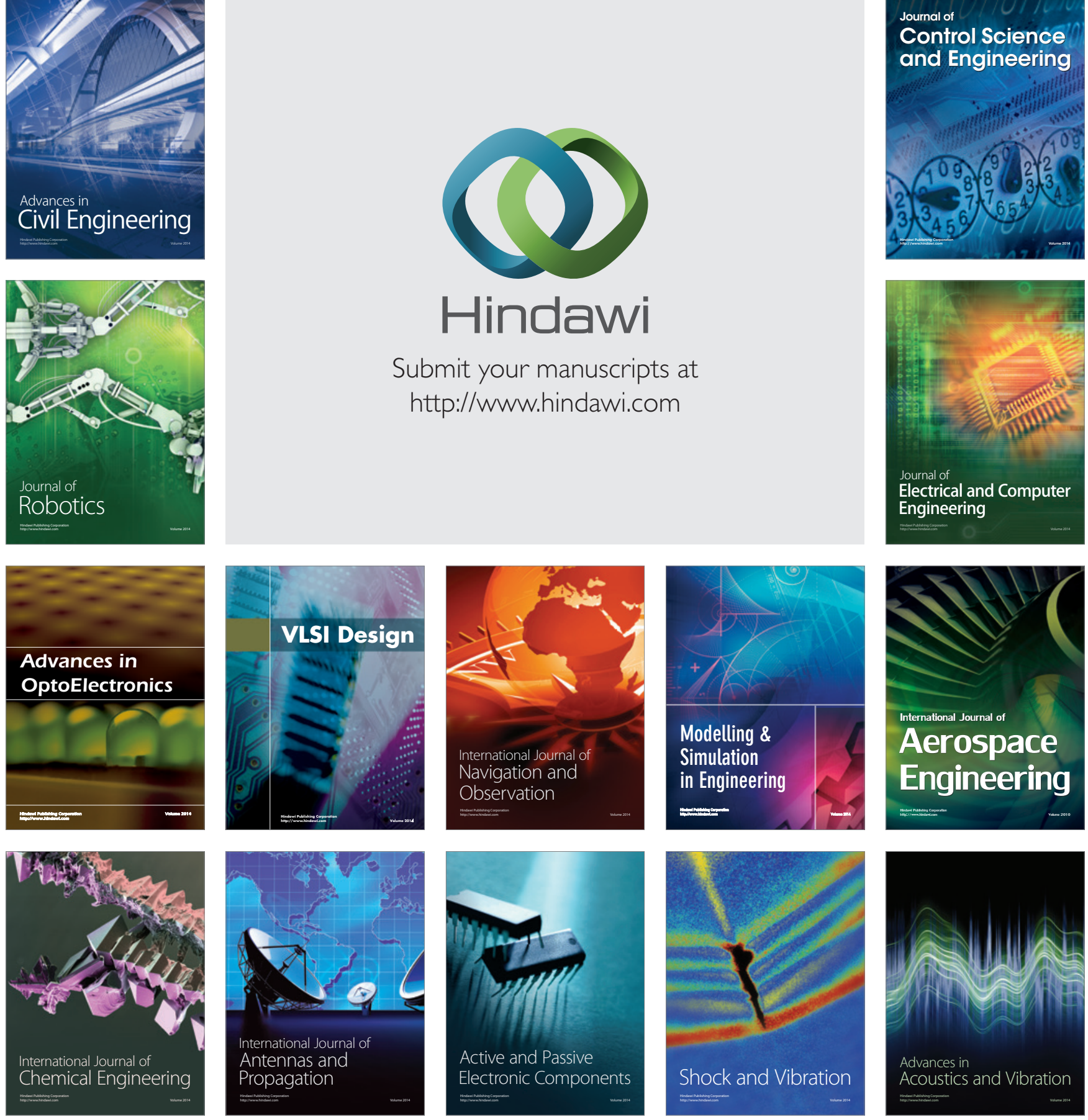